\title{
The Effect of Nursing Instructions on Nurses' Knowledge, Practice and Suggestions Regarding Adverse Events in Hemodialysis
}

\author{
Soheir Tawfeek Ahamed ${ }^{1, *}$, Salwa Abd El Gawad Sallam ${ }^{2, *}$ \\ ${ }^{1}$ Medical Surgical Nursing Department, Faculty of Nursing, Ain-Shams University, Egypt \\ ${ }^{2}$ Medical Surgical Nursing Department, Faculty of Nursing, Meonofia University, Egypt \\ *Corresponding author: dr.shaheen2015@hotmail.com; salwa_sallam@yahoo.com
}

\begin{abstract}
Hemodialysis now is an important and standardized treatment that is used as a life-saving treatment for more than 800,000 people worldwide who have end-stage renal disease. Protecting patients from adverse events at hemodialysis units is fundamental responsibility of all health team generally and nursing especially. Aim: To evaluate the effect of nursing instructions on nurses' knowledge, practice and suggestions regarding adverse events in hemodialysis. Research design: A quasi experimental design was utilized. Setting: The study was conducted in hemodialysis unit at King Khalid Hospital in Hail City; Kingdom of Saudi Arabia. Sample: Convenient sample of 26 nurses working in hemodialysis unit. Tools: First; Self-administered nurses' knowledge questionnaire; Second; Safety and quality observational checklist in hemodialysis session. Results: There was a statistical significant improvement between levels of nurses' knowledge and practice before and after nursing instructions, no relation between levels of nurses' knowledge and their levels of practice post nursing instructions and there was a difference in nurses' suggestion ranks regarding adverse events prevention pre and post nursing instructions. Conclusion: Implementation of nursing instructions regarding adverse events in hemodialysis was effective in improving the level of nursing knowledge and practice. Recommendations: Applying nursing instructions in other places with a large sample to enhance and confirm the current results.
\end{abstract}

Keywords: adverse events, hemodialysis nursing instructions, nurses' knowledge

Cite This Article: Soheir Tawfeek Ahamed, and Salwa Abd El Gawad Sallam, "The Effect of Nursing Instructions on Nurses' Knowledge, Practice and Suggestions Regarding Adverse Events in Hemodialysis.” American Journal of Nursing Research, vol. 6, no. 5 (2018): 237-243. doi: 10.12691/ajnr-6-5-4.

\section{Introduction}

The end stage of renal disease is defined as a complete irreversible failure of the kidney function and is a health problem that requires long-term and costly care. End stage of renal disease has reached an epidemic level which causing the greatest burden on health-care resources, a huge medical, social and economic problem for patients and their families [1].

Hemodialysis now is an important and a standardized treatment that is used as a life-saving treatment for more than 800,000 people worldwide who have end-stage renal disease [2]. In Egypt, the annual incidence of ESRD is about 74 per million, and the prevalence of patients on dialysis is 264 per million population [3]. Although effective hemodialysis and it gives patients hope for life indefinitely, there are many adverse events that may be life-threatening during it.

Hemodialysis centers are the most prone to adverse events occurrence due to various risk factors such as dialyzer errors, excess blood lose, patient falls, medication errors, inadequate infection control measures and faulty machine; Such events expose the patient to harm. The harm can be physical, social, psychological, including disease, injury, disability or death [4,5].

Adverse events (AEs) are incidents that occur during care delivery. Many studies in the United States, Canada, Australia, and England reported that 98,000 patients die each year from medical errors and preventable negative events occurred, while in our Arabic countries there are no documented ratios for adverse events [2]. Keeping patient's safety in the dialysis environment is a major concern for the patients and nurses. Therefore, improving the safety culture in the dialysis units is a prerequisite for reducing errors. For this reason, without attention to prevent errors during dialysis inevitably lead to adverse events [6,7].

Preventing (AEs) is a major challenge for quality improvement and enhancing nursing performance. Protecting patients from adverse events at hemodialysis units is a fundamental responsibility of all health team generally and nursing especially. Therefore, a consideration should be given to the importance of the suggestions that can be mentioned or opinions by the nursing team in preventing such adverse events [1,8]. Dialysis units should establish a dedicated safety team that improves patient outcomes. And since the dialysis nurses represent the largest component of the health care workforce, they are in a 
fundamental position to protect the patient and prevent adverse events by improving nursing performance and updating safety programs $[9,10]$.

Despite, the nurses who are working in hemodialysis centers should have knowledge and skills of adverse events to identify relevant risk factors and situations, looking for alternatives to reduce failure, adopting risk analysis methods and ensuring quality of care; but there is a noticeable increase in the incidence of such adverse events at dialysis sessions [11].

\subsection{Significant of the Study}

There is an urgent need for continuing education and training to improve the performance of nurses in dialysis units and to improve patient safety outcomes, thus contributing to the prevention of adverse events, which are reflected in decreasing mortality and morbidity rates and reducing economic burden on hospitals as well as patients. However, there is a lack of studies for evaluating the performance of nurses towards adverse events within renal dialysis units. Therefore, the researchers conducted this study which aims to evaluate the effect of nursing instructions on nurses' knowledge, practice and suggestions regarding adverse events in hemodialysis, hoping that this study will contribute to improve the nurses' quality performance and minimize the occurrence of adverse events within the dialysis unit.

\subsection{Aim of the Study}

This study aims to evaluate the effect of nursing instructions on nurses' knowledge, practice and suggestions regarding adverse events in hemodialysis.

\subsection{Research Hypotheses}

1. The level of nurses' knowledge and practice regarding adverse events in hemodialysis will significantly improve after implementing of nursing Instructions.

2. There will be a positive relation between nurses' knowledge and practice

3. There will be differences in nurses' suggestion ranks regarding adverse events prevention after implementing of nursing instructions.

\section{Subjects and Methods}

\subsection{Research Design}

A quasi experimental design was utilized to achieve the aim of the current study.

\subsection{Sampling and Population}

The study was conducted at hemodialysis unit in King Khalid Hospital in Hail City; Kingdom of Saudi Arabia. Convenient sample was used to collect 26 nurses, working at hemodialysis unit (in two shifts morning and after-noon) who present at the time of data collection, and welling to participate in the study. The data was collected from the beginning of April 2017 to the end of January 2018.

\subsection{Data Collection}

Two tools were used for data collection. Tool I: Self-administered nurses' knowledge questionnaire

This tool was developed by the researchers after reviewing of the related literature $[12,13]$. It was used to evaluate nurses' knowledge regarding adverse events at hemodialysis unit (pre \& post). The tool consists of the following:

a- Socio-demographic data such as: age, gender, level of education, years of experience \& attending training courses

b- Nurses' knowledge questionnaire which contains: i) general knowledge (20) questions related definitions of dialysis safety, patient safety, adverse events and hemodialysis complications. ii) Specific knowledge (22) questions covering different types, causes of adverse events and factors influencing adverse events; iii) nurses' actions taken if an adverse events occur (7). iv) Nurses' suggestions concerning the prevention of adverse events.

Scoring system (part i, ii and iii): The responses were given " 1 "for a correct answer and " 0 " for incorrect one to each question. Total score of $75 \%$ and more was considered satisfactory in knowledge while less than $75 \%$ was considered unsatisfactory. (High satisfactory level related to the importance and seriousness of knowledge in preventing the occurrence of adverse events).

Tool II: Observational Checklist:

Which containing Safety and Quality Checklist in Hemodialysis session: it is adapted from $[1,14]$ to assess level of nurse's practice regarding prevention of adverse events at hemodialysis. It contains 39 items divided into three levels. a) Pre session (11 items), b) during session (18 items), and c) post session (10 items)

Scoring system: The possible response for each item in observational checklist was met or not met. The scores given for each were 1 and zero respectively. Nurses were considered competent when the total score was more than $80 \%$ and nurses were considered not incompetent when the total score is less than $80 \%$.

Field work: The study was implemented during the period from the April 2017 to the end of January 2018. The researcher started by reviewing the related literature to gain more in-depth information about the subject, and able to design the appropriate data collection tools. This took about 2 months, and then content validity and reliability test were done before starting data collection process.

\subsection{Validity and Reliability}

Validity test was done by 5 experts from Medical Surgical Nursing specialty at Cairo and Port-said Faculty of Nursing. The nurses' knowledge questionnaire reliability was confirmed by Cronbach's alpha coefficient (alpha $=0.78$ for nurses' knowledge questionnaire). A pilot study was carried out on $10 \%$ of the total study sample before starting the data collection; to test the clarity, feasibility and applicability of the tools of the study. The necessary modifications were done after obtaining the results of the pilot study and expertise opinions with excluding the nurses included in the pilot study. 


\subsection{Ethical Considerations}

An official permission was obtained from the directions and chief person of the hospital and hemodialysis unit to conduct this study. The researchers explained the purpose of the study and their rights as study participants, including anonymity, confidentiality, and their rights to withdraw from the study at any time. Informed consent was obtained from the nurses who participated in the current study.

\subsection{Nursing Instructions Intervention}

Nursing instruction and intervention was performed through four phases as the following:

Assessment phase: The researchers assessed nurses' knowledge concerning adverse events at hemodialysis unit as a pre-test before the evaluation of their practice. The researchers gave the self-administered questionnaire to each nurse individually according to their available time and asked them to answer and fill it, about their knowledge concerning adverse events at hemodialysis unit and also asked them to write what they wanted and needed to know in relation to this subject; which involve the learners in the planning of the program and encouraging them to formulate their learning goals, which provides a flexible teaching focus on the learner's demands and not on the teacher's view of what the learners need to know. Then the researchers evaluated their practice through observational checklist.

Planning phase: Theoretical and practical nursing instructions were developed according to predetermined actual nurses' needs (pretest). They consisted of two parts (theoretical \& practical) as follows: Theoretical part: contains the following items; General knowledge regarding (definitions and hemodialysis complications). Specific knowledge concerned (types of adverse events, factors influencing adverse events, and what should happen if adverse events occur. And finally the suggestions of the nursing sample concerning prevention of adverse events. Practical part: demonstration and re-demonstration concerning safety and quality checklists in hemodialysis session.

Methods of teaching were presentation \& discussion by data show (computer) \& Handout.

Implementation phase: Through ten weeks in the morning and afternoon shifts and according the readiness of the studied nurses who were divided into four groups according to their available time. The theoretical content was two seminars for each group; its duration was 40-45 minutes. The content of theoretical part was given for all the studied nurses at the end of the last session. The practical part was one practical session for each group; its duration was 50-60 minutes

Evaluation phase: Examining the theoretical and practical instructions on studied nurses was started immediately after implementation of the nursing instructions (posttest) and using the same tools of the pretest. Then a comparison between the pre/posttests was done.

\subsection{Data Analysis}

The Statistical Package for Social Sciences (SPSS), Version 22.0 was used for data analysis. Data were presented using numbers, percentage, chi-square test and parried t Level of significant was thresholds at $\mathrm{p}<0.05$.

\section{Results}

Table 1. Demographic characteristics of the study sample $(\mathrm{N}=26)$

\begin{tabular}{|l|c|c|}
\hline Items & No & $\%$ \\
\hline Age: & & \\
$-<30$ & 7 & 26.9 \\
- 30-<40 & 19 & 73.1 \\
\hline Mean \pm SD & $30.32 \pm 3.11$ \\
\hline - Gender & & \\
- Female & 23 & 88.5 \\
- Male & 3 & 11.5 \\
\hline Language & & \\
- Arab & 8 & 30.8 \\
- Non-Arab & 18 & 64.2 \\
\hline Level of qualification: & & \\
- Diploma & 12 & 46.2 \\
- Bachelor & 14 & 53.8 \\
\hline Year of experience & & \\
- < 8years & 11 & 42.3 \\
- 8- <15 years & 15 & 57.7 \\
\hline Attended any training courses related to adverse events & & \\
- Yes & 9 & 34.6 \\
- No & 65.4 \\
\hline
\end{tabular}

Table 1 demonstrated that the Mean age of the studied sample was (30.3 \pm 3.11$)$, and $(88.5 \%)$ of them was female; also (64.2\%) of them they did not speak Arabic. Regarding the qualification (53.8 \%) had nursing Bachelor. In addition, (57.7 \%) of the studied sample had an experience from 8 to less than 15 years, and (65.4\%) of them didn't attend a training courses related to adverse events before.

Table 2 indicated that statistical significant improvement on nurses' knowledge at all items of general and specific toward adverse events of hemodialysis post intervention. Before intervention, (61.5\%\& $53.8 \%$ ) of the studied nurses reported unsatisfactory level of knowledge related to items of general knowledge and $(65.3 \%, 69.2 \%$, \& $76.9 \%$ ) related to items of specific knowledge respectively. After intervention, it was noted that the highest level of satisfaction concerning general knowledge was related to complications of hemodialysis while, concerning specific knowledge it was related to factors contributing to adverse events (100\% \& 76.9\%) respectively.

Table 3 illustrated that highly statistical significant improvement concerning the level of nurses' knowledge regarding all items of actions to take for adverse events at post instructional intervention. $(P<0.05)$. Before intervention; the lowest level of satisfactory knowledge was related to items that he/ she does if witnessed someone more senior than he/she caused an adverse events and what to do /say if a patient told him /her that he or she had made an error (34.6\% \& 38.5). After intervention, the highest level of satisfactory knowledge was related to items if adverse events occurred how she/he reports, whom to talk to if she / he caused an adverse events (100\%\&96.1\%) respectively. 
Table 2. Change of knowledge level for studied nurses toward adverse events of hemodialysis at pre \& post instruction intervention ( $\mathrm{N}=26$ )

\begin{tabular}{|c|c|c|c|c|c|c|c|c|c|c|c|}
\hline \multirow{3}{*}{\multicolumn{2}{|c|}{ Knowledge items }} & \multicolumn{4}{|c|}{ pre } & \multicolumn{4}{|c|}{ post } & \multirow{2}{*}{\multicolumn{2}{|c|}{ test }} \\
\hline & & \multicolumn{2}{|c|}{ Satis. } & \multicolumn{2}{|c|}{ Unsatis } & \multicolumn{2}{|c|}{ Satis. } & \multicolumn{2}{|c|}{ Unsatis } & & \\
\hline & & No & $\%$ & No & $\%$ & No & $\%$ & No & $\%$ & $X^{2}$ & $P$ \\
\hline \multicolumn{12}{|c|}{ General knowledge } \\
\hline 1 & Definition of patient safety & 10 & 38.5 & 16 & 61.5 & 24 & 92.3 & 3 & 11.5 & 23.3 & 0.05 \\
\hline 2 & Complications of hemodialysis & 12 & 46.1 & 14 & 53.8 & 26 & 100 & 0 & 0 & 22.7 & 0.05 \\
\hline \multicolumn{2}{|c|}{$\begin{array}{l}\text { Total } \\
\text { Mean } \pm \text { S.D }\end{array}$} & \multicolumn{4}{|c|}{$36.67 \pm 8.01$} & \multicolumn{4}{|c|}{$93.32 \pm 11.58$} & \multicolumn{2}{|c|}{$\begin{array}{l}\text {-test } \\
20.05 \\
\end{array}$} \\
\hline \multicolumn{12}{|c|}{ Specific knowledge } \\
\hline 1 & Types of adverse events & 9 & 34.6 & 17 & 65.3 & 21 & 19.2 & 5 & 19.2 & 27.2 & 0.05 \\
\hline 2 & Causes of adverse events & 8 & 30.7 & 18 & 69.2 & 24 & 92.3 & 2 & 7.7 & 16.4 & 0.05 \\
\hline 3 & Factors contributing to adverse events & 7 & 26.9 & 20 & 76.9 & 23 & 88.5 & 3 & 11.5 & 15.9 & 0.05 \\
\hline \multicolumn{2}{|c|}{$\begin{array}{l}\text { Total } \\
\text { Mean } \pm \text { S.D }\end{array}$} & \multicolumn{4}{|c|}{$41.74 \pm 5.69$} & \multicolumn{4}{|c|}{$89.65 \pm 19.36$} & \multicolumn{2}{|c|}{$\begin{array}{c}1 \\
\text {-test } \\
32.1 \\
\end{array}$} \\
\hline
\end{tabular}

Table 3. Change the level of nurses' knowledge regarding Actions to take for adverse events at pre \& post instruction intervention $(\mathrm{N}=\mathbf{2 6})$

\begin{tabular}{|c|c|c|c|c|c|c|c|c|c|c|}
\hline \multirow{3}{*}{ statement } & \multicolumn{4}{|c|}{ pre } & \multicolumn{4}{|c|}{ post } & \multicolumn{2}{|c|}{ test } \\
\hline & \multicolumn{2}{|c|}{ Satis. } & \multicolumn{2}{|c|}{ Unsati. } & \multicolumn{2}{|c|}{ Satis. } & \multicolumn{2}{|c|}{ Unsati. } & \multirow{2}{*}{$\mathbf{X}_{2}$} & \multirow{2}{*}{$\mathbf{P}$} \\
\hline & No & $\%$ & No & $\%$ & No & $\%$ & No & $\%$ & & \\
\hline 1. I would know what to say if $\mathrm{i}$ caused an adverse events & 13 & 50 & 13 & 50 & 23 & 88.5 & 3 & 11.5 & 15.43 & $<0.05$ \\
\hline 2. I would know who to talk to if $i$ caused an adverse events & 11 & 42.3 & 15 & 57.7 & 25 & 96.2 & 1 & 3.8 & 19.20 & $<0.05$ \\
\hline $\begin{array}{l}\text { 3. I would know what to do if i witnessed someone more senior } \\
\text { than me caused an adverse events }\end{array}$ & 9 & 34.6 & 17 & 65.4 & 22 & 84.6 & 4 & 15.4 & 35.90 & $<0.05$ \\
\hline $\begin{array}{l}\text { 4. I would know what to do if someone in may health team } \\
\text { caused an adverse events }\end{array}$ & 15 & 57.7 & 11 & 42.3 & 24 & 92.3 & 2 & 7.7 & 21.33 & $<0.05$ \\
\hline $\begin{array}{l}\text { 5. If I caused an adverse events I would to discuss it with } \\
\text { someone }\end{array}$ & 14 & 53.9 & 12 & 46.1 & 23 & 88.5 & 3 & 11.5 & 12.79 & $<0.05$ \\
\hline $\begin{array}{l}\text { 6. I would know what to do /say if a patient told me i had made } \\
\text { an error. }\end{array}$ & 10 & 38.5 & 16 & 61.5 & 24 & 92.3 & 2 & 7.7 & 18.17 & $<0.05$ \\
\hline 7. If adverse events occurred I would want how to report. & 12 & 46.1 & 14 & 53.9 & 26 & 100 & 0 & 0 & 22.13 & $<0.05$ \\
\hline \multirow{2}{*}{$\begin{array}{l}\text { Total } \\
\text { Mean } \pm \text { S.D }\end{array}$} & \multirow{2}{*}{\multicolumn{4}{|c|}{$13.24 \pm 5.41$}} & \multirow{2}{*}{\multicolumn{4}{|c|}{$22.19 \pm 7.34$}} & \multicolumn{2}{|c|}{ t-test } \\
\hline & & & & & & & & & 16.4 & $<0.05$ \\
\hline
\end{tabular}

Table 4. Change of practice competence level for studied nurses toward adverse events of hemodialysis pre \& post instruction intervention $(\mathrm{N}=\mathbf{2 6})$

\begin{tabular}{|c|c|c|c|c|c|c|c|c|c|c|}
\hline \multirow{3}{*}{ items } & \multicolumn{4}{|c|}{ pre } & \multicolumn{4}{|c|}{ post } & \multirow{3}{*}{ test } & \multirow{3}{*}{$p$} \\
\hline & \multicolumn{2}{|c|}{ Comp. } & \multicolumn{2}{|c|}{ Incomp. } & \multicolumn{2}{|c|}{ Comp. } & \multicolumn{2}{|c|}{ Incomp. } & & \\
\hline & No & $\%$ & No & $\%$ & No & $\%$ & No & $\%$ & & \\
\hline 1. Pre Session & 11 & 42.3 & 15 & 57.7 & 25 & 96.2 & 1 & 3.8 & \multirow{3}{*}{19.2} & \multirow{3}{*}{$<0.05$} \\
\hline Mean \pm S.D & \multicolumn{4}{|c|}{$7.08 \pm 1.80$} & \multicolumn{4}{|c|}{$11.88 \pm 1.16$} & & \\
\hline 2. during Session & 5 & 19.2 & 21 & 80.8 & 23 & 88.5 & 3 & 11.5 & & \\
\hline Mean \pm S.D & \multicolumn{4}{|c|}{$8.44 \pm 2.2$} & \multicolumn{4}{|c|}{$14.36 \pm 1.68$} & 22.3 & $<0.01$ \\
\hline 3. Post Session & 6 & 23.1 & 20 & 76.9 & 24 & 92.3 & 2 & 7.7 & & \\
\hline Mean \pm S.D & \multicolumn{4}{|c|}{$4 \pm 1.04$} & \multicolumn{4}{|c|}{$8.04 \pm 0.78$} & 14.7 & $<0.01$ \\
\hline $\begin{array}{l}\text { Total } \\
\text { Mean } \pm \text { S.D }\end{array}$ & \multicolumn{4}{|c|}{$14 \pm 2.82$} & \multicolumn{4}{|c|}{$31.2 \pm 2.81$} & \multicolumn{2}{|c|}{$\begin{array}{c}\text { t-test } \\
28.6\end{array}$} \\
\hline
\end{tabular}

Table 4 showed that there was a statistical significant improvement regarding nursing practice competence occurs at post instructional intervention at all items (pre, during and post session of hemodialysis). ( $P<0.05)$.

Table 5. Correlation between total level of knowledge and total competent level of practice among studied nurses $(\mathrm{N}=26)$

\begin{tabular}{|cc|c|c|}
\hline \multirow{2}{*}{ Practice } & \multirow{2}{*}{ Knowledge } & \multicolumn{2}{|c|}{ test } \\
\cline { 3 - 4 } & pre & 0.051 & $P$ \\
\hline I. & post & 0.164 & $<0.05$ \\
\hline II. & pren & $<0.05$ \\
\hline
\end{tabular}

Table 5 discovered that there no correlation between total level of knowledge and total competence level of practice pre and post intervention $(r=0.051 \& 0.164)$ respectively.

Table 6 indicated a variation in the nurses' ranking concerning the most important suggestions to prevent adverse events at hemodialysis unit pre and post intervention. Concerning the health facility service suggestion ranking, before intervention the first three suggestions ranking were reducing an excessive workload and the number of patients, provided a unit for continuing education \& improved communication skills between 
health team workers, then after intervention changed to reduce excessive workload and the number of patients, provided a unit for continuing education and provided an adequate human and material resources. As shown in the same table, the first two suggestions ranking concerning to health team and patients before intervention were assessed health conditions of the health team and guidance to the health team and patients. While the ranking changed after intervention to be a suggestion to assess health conditions of the health team and activating the justice system in the reward and punishment.

Table 6. Nurses' ranking regarding the most important suggestions to prevent adverse events at hemodialysis unit post intervention

\begin{tabular}{|l|c|c|c|c|}
\hline \multirow{2}{*}{ Suggested items } & \multicolumn{2}{|c|}{ pre } & \multicolumn{2}{|c|}{ post } \\
\cline { 2 - 5 } & $\%$ & Rank & $\%$ & Rank \\
\hline $\begin{array}{l}\text { I. Concerning to the health facility } \\
\text { service } \\
\text { - Reduce excessive workload and number } \\
\text { of patient }\end{array}$ & 92.3 & 1 & 100 & 1 \\
\hline $\begin{array}{l}\text { - Provide a unit for continuing education. } \\
\text { - Provide adequate human and material } \\
\text { resources. }\end{array}$ & 78.5 & 2 & 96.1 & 2 \\
\hline $\begin{array}{l}\text { - Improve communication skills between } \\
\text { health team work }\end{array}$ & 80.8 & 3 & 80.7 & 4 \\
\hline $\begin{array}{l}\text {-Periodic follow-up with the correction } \\
\text { plan }\end{array}$ & 65.4 & 5 & 76.9 & 5 \\
\hline $\begin{array}{l}\text { II. Concerning to health team and } \\
\text { patients }\end{array}$ & & 84.6 & 3 \\
\hline $\begin{array}{l}\text { - Assess health condition of the health } \\
\text { team. }\end{array}$ & 88.5 & 1 & 92.3 & 1 \\
\hline $\begin{array}{l}\text {-Activating the justice system in reward } \\
\text { and punishment. }\end{array}$ & 69.2 & 4 & 88.5 & 2 \\
\hline $\begin{array}{l}\text { - Guidance to the health team and patients } \\
\text { goals }\end{array}$ & 80.8 & 2 & 80.7 & 3 \\
\hline $\begin{array}{l}\text {-Compliance of the health team to safety } \\
\text { goals }\end{array}$ & 73.1 & 3 & 73.1 & 4 \\
\hline
\end{tabular}

\section{Discussion}

Adverse events (AEs) were incidents that occurred during care provision resulted in harm to the patient. These events may occur in a variety of health facility services, including hemodialysis units. The recent study conducted in the United States of America estimated that more than 400,000 patients died because of AEs, pointing to the urgency for changes and improvements in patient safety [15]. The dialysis nurse is a key element in the health team providing a direct health care to these patients and represents the first line of defense in prevention of such events through an appropriate nursing management care in hemodialysis sessions. [16,17] Therefore, the hemodialysis nursing care is still a need to implement the best guidelines into clinical practice as the process to patients' safety [9]. So the aim of the current study was to evaluate the effect of clinical nursing instructions on nurses' knowledge and practice regarding adverse events in hemodialysis.

As regarding nurses' knowledge towards (AEs) of hemodialysis, the current study revealed that the studied nurses had unsatisfactory levels towards all items of general and specific knowledge before intervention. While there was a statistical significant improvement in their level of knowledge at all items post intervention as indicated by higher total mean scores on the posttest $92.15 \pm 7.06$. This result may be the positive effect of nursing instructions, this possibility is supported by about two-thirds of the studied sample did not attend an educational program regarding the adverse events of hemodialysis before. This proves the importance of the continuity of such educational training programs. In the study conducted by [1] confirmed that hemodialysis is a lifesaving treatment for a patient with renal failure, but if the nurses didn't understand the concept of hemodialysis patient safety and its complications, it becomes a serious risk to life threat to the patient.

On the other hand, these results correspond with many studies [18,19], who stressed that hemodialysis nurse, should have knowledge of types and causes of adverse events to identify related risks and situations that affected the patient safety and led to prevent patient harm and ensure the quality of care. Also, [20], stressed in his report that providing safe care to the patient that depends on nurses' qualifications to detect factors which contributed to adverse events occurrence.

With respect to nurses' knowledge of actions to be taken if adverse events occur, the current findings found that there were statistically significant improvement in their level of knowledge in the post instructions of intervention. Where all of them were able to report if adverse events occur also, the majority of them have realized whom they are talking to if they had adverse events, what to do if someone in health team caused adverse events and they know also what to do /say if a patient told them that they made an error. This result may be one of the important strength points for the instruction intervention as it contributed to clarify how the nurse took actions correctly in the adverse events which also led to reduce the risk of these events and also help in maintaining the safety of the patient.

This vision coincided with both [10,21], who revealed in their study the increase of the nurse's knowledge and awareness of the correct action in the adverse events occur which is reflected on prevention and reduction of the patient's hazards and contributed to improve the safety during hemodialysis sessions. Also, [17] added that the timely detection of situations that can prompt AE essential to understand its causes, calculate the risks for corrective measures. From another point of view, [22,23] stressed in their study the increase of the nurse's awareness of how to take properly action towards adverse events if occur, increases his/her self-confidence which reflected positively on the quality of care provided and patient outcome.

Regarding practical competence level of nurses, in general the current finding pointed out that there was a statistical significant improvement before and after practice part in the instructions' intervention over the different times of the dialysis session. This finding is consistent with the results of a similar recent study conducted by [1].

However, with a careful observation of the current results, they revealed a variation in the level of improvement of nurses' competence, as the highest improvement during and post-session period while the 
lowest improvement towards the pre-session period. This can be attributed to the fact that the nurses had an intermediate competence in the pre-session practical steps because most of clinical steps in pre session based on a routine care. But they needed a further instruction and a training towards steps during and post-session because most adverse events occur during these sessions. This result is supported by $[24,25]$ who stressed that the lack of competence and practical training in nursing contributed directly to the increase of adverse events occurred for the hemodialysis patients, especially during the session, because of complications that occur. Also, [26] emphasized that the training of nurses is a cornerstone of positive impact on patient outcomes.

Regarding the correlation between the total of nurses' knowledge and total level of practical competence, the current finding discovered that there was no correlation (total knowledge and practice) pre and post intervention ( $r=0.051 \& 0.164)$ which gave a reflection that each variable was independent. This finding may be due to around one third of the study sample was attended training courses before which affected the level of their experiences than the level of knowledge. This result was consistent with the result of study conducted by [27] who explained in his study, that improvements in the practical performance of nurses did not depend on their level of knowledge but they based on practical training and refreshment of their experience. In contradictory with our results are [28,29,30,31] confirmed that there was a relationship between improvement the level of nurse's knowledge and improvement of their practices. Through this result we noted that it did not support the second hypothesis of the current study.

The current study concerned with comparing the ranking of the nurses' suggestions on how to prevent adverse events in hemodialysis unit pre and post clinical instructions' intervention because they are the first line of defense in preventing it. This supported by [32] who recommended in his study that they should know the views and suggestions of the caregivers on how to prevent and reduce the occurrence of negative events within the departments they work because they are the real vision within their work units.

From this point, the current finding discovered changes in the ranking before and after intervention. Before intervention, most of the studied nurses ranking the first three suggestions to prevent adverse events concerning with the health service as the following; reduce excessive workload and number of patients, provide a unit for continuing education and improve communication skills between health team work. After intervention, there were no changes in the rank of the first and second suggestions but the majority of studied sample replaced the third suggestion by providing adequate human and material resources. This result reflects the nurses' awareness towards the role of educational programs in preventing the occurrence of adverse events and also emphasizing that the shortage of nurses are the most important factors causing such events which to be a main cause of death in hospitalized patients. These results correspond to both $[8,30]$, who stressed that the shortage of nurses and increased work load contributed greatly to the occurrence of errors; also the lack of educational programs directly affected the quality of health care provided.

As the same line table discovered that concerning nurses' suggestion regarding to heath team and patients before intervention; the most of the nurses ranking the first two suggestions to prevent adverse events as the following; Assess health condition of the health team and guidance to the health team and patients. While the ranking was following after the intervention of the clinical instructions, the first suggestion did not change its rank than pre intervention but the second suggestion was mostly of studied sample confirmed to be activated the justice system in reward and punishment. In recent study conducted by [29], recommended that the policy of reward and punishment should be applied as a way to improve the performance of nurses and also as a means of preventing AE.

Finally, [33,34] added that the nurses' suggestions in preventing the occurrence of the negative events are a cornerstone because they depend on the real need of the nurses, to develop their performance and qualifications.

\section{Conclusion}

Implementation of nursing instruction regarding adverse events in hemodialysis was effective in improves level of nurses' knowledge and practice and, there was a difference in nurses' suggestions ranking regarding adverse events prevention pre and post intervention and there was a difference in nurses' suggestions ranking regarding adverse events prevention pre and post nursing instruction intervention.

\section{Recommendations}

Based on the study finding the study recommended to apply the program in other places and with a large sample to enhance and confirm the current results. Follow up on nurses' and other health team suggestions with regards to prevention of adverse events and their placement in the safety plan of the hemodialysis units. Activating the policy of reward and punishment among the members of the health team

\section{Limitations}

Small numbers of the sample in present study. As well different cultures and language between the study sample, which negatively affected on their communication skills.

\section{References}

[1] Dawood R., El-sebai., Salem Y and Hussein R. (2016): Effect of implementing a protocol of nursing care on hemodialysis patients' safety outcomes. IORS Journal of nursing and Health Science. Sep-Oct.2016; Vol 5; (5).

[2] Silver S., Thomas A., Rathe A., Robinson P., Wald \& R et al. (2015): Development of a hemodialysis safety checklist using a structure panel process. Clin kidney J. 2015 Feb 12 (2)5. 
[3] Lotfy E, Sakla N, El-Naggar S. Demographic study with risk factors of hemodialysis patients in Damietta Governorates, Egypt. International journal of Advanced Research (2015). Vol 3, Issue 10, 91-100.

[4] Moursy A. \& Sharaf A.(2017): Vascular access care at hemodialysis unit; nurses compliance to infection prevention and control practices. IORS Journal of Nursing and Health Science. Mar -Apr.2017; Vol. 6, Issue 2.

[5] Thomas-Hawkins, C., \& Flynn L. (2013). Patient safety culture and nurse-reported adverse patient events in outpatient hemodialysis facilities. Nephrology Nursing JOURNAL. 40(2), 170.

[6] Thomas- Hawkins C.(2014): Culture of patient safety in dialysis care. Culture of patient safety in dialysis care Renal Society of Australasia Journal, 10(2). 86-90.

[7] Garrick R., Kliger A. and Stefanchik B. (2012). Patient and facility safety in hemodialysis: opportunities and strategies to develop a culture of safety. Clinical Journal of the American Society of Nephrology, Apr; 7(4): 680-688.

[8] Minn W.(2014): Enhancing patient safety in hemodialysis nursing care practice among nurses in the renal unit at the defense services general hospital: a best practice implementation project. JBI Database of Systematic reviews \& implementation reports. 2014; 12(9).

[9] Alamry A., Al Owais S., Al-Dorzi.,\& Taher S.(2012): patient saf. Incidence reporting at a tertiary care hospital in Saudi Arabia. J. patient Saf., 2(8): 81-87.

[10] Hassan S.,Sorour A., Eldahshan K, and Elmoghazy G. (2013). Nurses knowledge and practice regarding intradialytic complication for hemodialysis patient. Journal of American Science; 9(11): 300-307.

[11] Thomas- Hawkins., Flynn L. \& Weaver S. (2015). Nurse Manager Safety practices in outpatient hemodialysis units. Nephrology nursing journal Mar/Apr 2015: 125-133.

[12] UNIVERSITY OF TAMPERE Health Care Professionals' Knowledge and Attitudes Regarding Patient Safety and Skills for Safe Patient Care. Acta. Tampere 2016, University of Tampere, School of Health Sciences Finland

[13] Almaramhy H., Al-Shobaili H., El-Hadary K. and Dandash K. (2011). Knowledge and attitude towards patient safety among a group of undergraduate medical students in Saudi Arabia. IJHS international journal of health sciences (Qassim). 2011 Jan; 5(1): 59-67.

[14] Marcelli D.,Matos A., Sousa F., Peralta R. \&et al. (2015) Implementation of a quality and safety checklist for hemodialysis sessions. Clin Kidney J. 2015 Jun; 8(3).

[15] World Health Organization (WHO). Conceptual framework for the international classification for patient safety. Taxonomy. World Alliance for patient safety. 2009[cited in 2016 Feb19]. Available from: http://www.who int/patientsafety/taxonomy/icps_fuul-report.pdf.

[16] Vafaei A., \& Nobahar M. The care preference of patients under hemodialysis. J. Ren. Inj. Prev. 2017, 6. 210-215.

[17] Sousa M., Camargo A., Bezerra A., Freitas J. and Neves G. \& et al (2016): Prevalence of adverse events in a hemodialysis unit. Capa v.24, N.6.

[18] Thomas A., Silver S., Rathe A., Robinson P., Wald P., \& et al (2016): Feasibility of a hemodialysis safety checklist for nurses and patients: a quality improvement study. Clin kidney J. $2016 \mathrm{Ju}$; 9(3): 335-342.

[19] Sousa M., Camargo A., Bezerra A., Freitas J. and Miasso A. (2013). Adverse events in hemodialysis: reports of nursing professionals. Revista da Escola de Enfermagem da USP. Feb Vol. 47. No.1.

[20] Shahdadi H. \& Rahnama M. (2018). Experience of nurses in hemodialysis care:A phenomenological study. J. Clin. Med. 2018; (7) 30 .

[21] Bray B \& Metcalfe W.(2015): Improving patient safety in hemodialysis. Clin kidney J. 2015 Jun; 8 (3): 262-264.

[22] Dziubek W. the level of anxiety and depression in dialysis patients undertaking regular physical exercise training. Kidney and blood pressure research journal.2016; 4(1): 86-88.

[23] Mohesn M., Mohammed W., Riad N and Atia G. (2013). Impact of designed nursing intervention protocol for hemodialysis patients on patients' outcomes. Journal of Biology, Agriculture and healthcare. Vol.3, No.16, 2013:53-56.

[24] Hamed O. (2016). Knowledge and Practice of Nurses towards Care of Hemodialysis Patients at Governmental Hospitals in Khartoum State. thesis submitted in Partial fulfillment for the requirement of M.Sc Degree in Health Management. The National Ribat University Faculty of Graduate Studies \& Scientific Research.

[25] Mabrouke E. Assessment of hemodialysis adequacy in patients with chronic kidney disease in the hemodialysis unit at Tanta University Hospital in Egypt. Kasr Al-Ainy Medical Journal. 2015; 21(2): 47-54.

[26] Pageb M. Practice implications of keeping patients safe. Journal of Care Quality. 2014; 20(3): 193-197.

[27] Paranagua T., Bezerra AL., Silva A., \&Filho A.(2016): Prevelance of no harm incidents and adverse events in a surgical clinic. Acta Paul Enferm. [citaded in 2016 Feb 19]; 26(3): 256-262. Available from: http://www.scielo.br/pdf.

[28] Ulrich B., \& Kear T. (2014): Patient Safety Culture in Nephrology Nurse Practice Settings: Initial Findings. Nephrology Nursing Journal Sep.-Oct. 2014 Vol. 41, No. 5.

[29] Ahamed S \& Ebraheim M. (2017): Glascow Coma Scale Technique: Effect of theoretical and practice educational program on Nurses' compliance. IORS Journal of Nursing and Health Science. May -June.2017; Vol. 6, Issue 3.

[30] Bassuni E. and Bayoumi M.(2015): Improvement critical care patient safety: Using nursing staff development strategies, at Saudi Arabia. Global Journal of Health science; Vol.7, No.2; 2015: 335-341.

[31] Eid N. \& Abd el-Aziz.2013): Proposed Developed Standards: Staff Nurses Compliance at Dialysis Unit. Greener Journal of Medical Sciences ISSN: 2276-7797 Vol. 3 (5), pp. 179-189, July 2013.

[32] Kliger A.(2015): Maintaining safety in the dialysis facility. Clin J Am Soc Nephrol. 2015 apr.7; 10(4): 688-695.

[33] Mendes W., Pavao ALB., Matins M., Travassos C. The feature of preventable adverse events in hospital in the State of Rio de janeiro, razil. Rev Assoc Med Bars.[ cited in 2016 Feb19]; 59(5): 421-428.

[34] Lira A. \& Oliveira L. (2011): nursing diagnosis: educational strategy based on problem-based learning. Rev. Latino-am enfermagem July -Aug. Vol. 19 (4). 